\title{
Blind Known Interference Cancellation
}

\author{
Shengli Zhang, Member, IEEE, Soung-Chang Liew, Fellow, IEEE, and Hui Wang, Member, IEEE
}

\begin{abstract}
This paper investigates interference-cancellation schemes at the receiver, in which the interference data, which is valid data intended for another receiver, is known a priori. The interference channel, however, is unknown (the blind part). Such a priori knowledge is common in wireless relay networks. For example, a relay could be relaying data that was previously transmitted by a node $A$. If node $A$ is now receiving a signal from another node $B$, the interference from the relay is actually self-information known to node $A$. Besides the case of selfinformation, the node could also have overheard or received the interference data in a prior transmission by another node. Directly removing the known interference requires accurate estimate of the interference channel, which may be difficult in many situations. In this paper, we propose a novel scheme, Blind Known-Interference Cancellation (BKIC), to cancel known interference without interference channel information. BKIC consists of two steps. The first step combines adjacent symbols to cancel the interference, exploiting the fact that the channel coefficients are almost the same between successive symbols. After such interference cancellation, however, the signal of interest is distorted. The second step recovers the signal of interest amidst the distortion. We propose two algorithms for the critical second steps. The first algorithm (BKIC-S) is based on the principle of smoothing. It is simple and has near optimal performance in the slow fading scenario. The second algorithm (BKIC-RBP) is based on the principle of real-valued belief propagation. Since there is no loop in the Tanner graph, BKIC-RBP can achieve MAP-optimal performance with fast convergence, and has near interference-free performance even in the fast fading scenario. Both BKIC schemes outperform the traditional self-interference cancellation schemes that have perfect initial channel information by a large margin, while having lower complexities.
\end{abstract}

Index Terms-Self-interference, Blind, MAP, Belief Propagation, Interference Cancellation.

\section{INTRODUCTION}

$\mathbf{T}$ HE use of relay in wireless networks is attracting increasing attention $[1,2]$ because of the many advantages it brings, such as improved connectivity and reduced power consumption. Many multi-hop relay standards, including $802.16 \mathrm{j}, 802.11 \mathrm{~s}$, are being developed.

In wireless relay networks, a node may receive a target signal intended for it superimposed with interference. In many scenarios, the receiver actually knows the data contained in the interference [3], either because the interference was signal previously received/overheard by the node, or the interference is self-information previously transmitted by the node and now relayed by a relay to another node. One example of known interference is when physical-layer network coding over infinite field [4] (e.g., analog network coding [5]) is used

S. Zhang and H. Wang are with the Shenzhen MCSP Key Lab, Department of Communication Engineering, Shenzhen University, Shenzhen, China. email:(zsl,wanghsz)@gmail.com .

S. Liew is with the Department of Information Engineering, the Chinese University of Hong Kong, HK, China,.

Manuscript received April 19, 2005; revised January 11, 2007. in a two-way relay channel, as shown in Fig. 1a. Another example is a linear-chain one-way relay network [6], as shown in Fig. 1b. Many other scenarios of known interference can be found in [3].

The method to deal with known interference is straightforward in theory. The receiver first estimates the channel coefficient of the interference signal and then removes the known interference from the received signal [3]. In this paper, we refer to this scheme which requires the knowledge (and therefore the estimation) of the interference channel as traditional KIC (Known Interference Cancellation). This scheme does not perform well when the channel estimation is inaccurate.

Accurate channel estimation is non-trivial even in the absence of interference. This is the reason why non-coherent detection schemes that do not require channel information are still widely studied and used in wireless communication systems [7]. In the presence of interference, channel estimation faces additional challenges. First, channel estimation is more difficult because the training sequences are corrupted by the superposition of two signals. Simultaneous estimation of the channel coefficients of the target signal and the interference can be complex. For example, when the two training sequences are the same and they overlap with each other, we can only obtain the summation of the two channel coefficients but not their individual values. Second, when the power of the interference is much larger than that of the target signal, a tiny estimation error of the interference channel may cause the interference cancellation process to leave behind a relatively large residual interference with respect to the target signal. Third, it may be impossible to estimate the channel accurately [8] in mobile environment with fast fading. The channel estimated from the training sequence may have changed by the time the data is received.

We note that the above difficulties apply regardless of whether the interference is known or unknown: for both cases, the training sequences are presumed known in order to enable channel estimation.

There has been some work trying to tackle the problem of estimating the channels of two superimposed packets. For example, in physical-layer network coding [9-11], the channels of two superimposed packets need to be estimated. To deal with the channel estimation problem, the physicallayer network coding implementation in [12] uses orthogonal sequences for the two packets. The analog network coding scheme in [5], on the other hand, attaches the training sequence to both the front end and back end of a packet, and time the transmissions of the two packets so that one of them has interference-free front end and the other one has interferencefree back end. These schemes use new frame designs and are not compatible with legacy wireless systems. Ref. [13] uses an optimization scheme to estimate the channels with two 
specially designed training sequences. The estimation accuracy is much poorer than single-channel estimation. Non-coherent ANC schemes that avoid channel estimation have also been studied $[14,15]$. However, these non-coherent schemes suffer from SNR degradation of about $3 \mathrm{~dB}$ compared with the coherent schemes. By contrast, the blind known-interference cancellation (BKIC) schemes proposed in this paper can obtain near-perfect performance - specifically, performance close to that of a point-to-point communication link without interference - while avoiding estimation of the interference channel.

BKIC has three advantages over the traditional methods: 1) good performance; 2) no need for interference channel estimation; and 3) compatibility with legacy systems. The principle on which BKIC operates is based on the observation that the wireless channel typically remains almost unchanged between adjacent symbols [8]. BKIC uses the interference in one symbol to cancel the interference in its adjacent symbol. For example, if the interference channel is $h$ and the interference symbol is 1 , then the interference in the current symbol is $h$. If the interference data in the adjacent symbol is -1 , then the corresponding known interference is approximately $-h$. The interference can be cancelled with each other if we combine the two symbols. Such adjacent-symbol combination, however, may result in distortion of the target signal. Thus, a key issue is how to remove such distortion as the next step. We propose two schemes, smoothing (BKIC-S) and real-valued belief propagation (BKIC-RBP), to equalize the resulting distortion.

This paper considers BKIC schemes for both flat fading channel and frequency selective channel. We show that the performance of our schemes is almost the same as that of a pure coherent point-to-point channel without interference (note: this is a theoretical upper bound for our system). Besides its excellent performance, our schemes are also attractive because of their low complexity and compatibility with legacy systems. Specifically, our schemes do not require special changes to the frame structure or the operation of the transmitter. A salient feature of our schemes is that they could be realized by an add-on module inserted into the signal processing path of the receiver without requiring complicate modifications to the existing module.

The remainder of this paper is organized as follows. In section II, we present the system model and architecture of BKIC. Section III explains BKIC under the flat fading channel assumption, and Section IV extends the discussion to the frequency selective fading channels. In Section V, we analyze the performance. We validate and supplement the analytical results with numerical simulation in Section VI. Finally, Section VII concludes this paper.

\section{System Model AND ARChitecture}

\section{A. System Model}

Known interference is common in many wireless networks. Two Way Relay Channel (TWRC) with analog network coding [5] in Fig. 1a, and one-way relay chain network in Fig. 1b, are two examples with known interference. In this section,
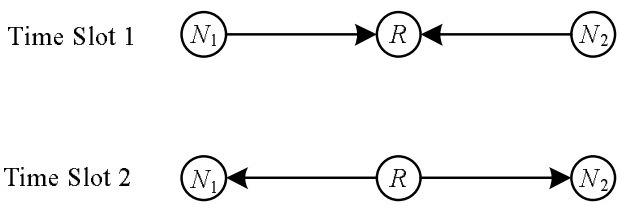

(a) Two way relay with analog network coding, where the two sources $\mathrm{N} 1$ and $\mathrm{N} 2$ transmit simultaneously to the relay in the first time slot, and the relay amplifies and broadcasts the received signal to both sources in the second time slot.

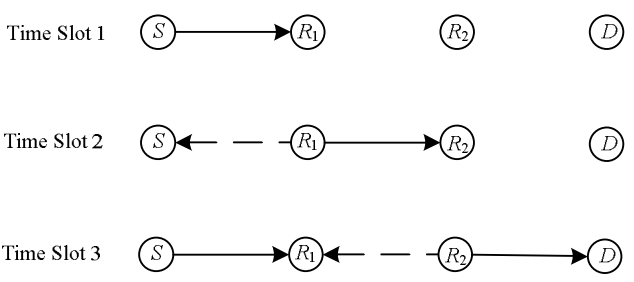

(b) One way relay channel in a chain.

Fig. 1: Two wireless networks with known interference.

we present the general mathematical formulation for known interference systems. For a focus, consider the chain network in Fig. 1b. The source node $S$ transmits to the destination node $D$ through two relay nodes, $R_{1}$ and $R_{2}$, and there are no cross-hop transmissions.

For simplicity, we assume one dimensional $q$-ary ASK modulation at all the nodes; our method can be easily extended to other modulations, including two-dimensional modulations. As shown in Fig. 1b, in time slot $1, S$ transmits a packet to relay $R_{1}$; in time slot $2, R_{1}$ forwards it to the second relay $R_{2}$; and in time slot $3, R_{2}$ forwards it to destination $D$, while node $S$ sends a new packet to $R_{1}$ at the same time. The transmissions in time slots 2 and 3 are repeated for the delivery of successive packets from $S$ to $D$.

Note that node $R_{1}$ receives a superposition of the two packets, one from $S$ and one from $R_{2}$. With the assumption of symbol level synchronization [16], the $k$-th received symbol at $R_{1}$ can be expressed as

$$
r(k)=\sum_{l=0}^{L_{x}} h_{x}(k, l) x(k-l)+\sum_{l=0}^{L_{I}} h(k, l) I(k-l)+n(k)
$$

where $x(k) \in A=\{-q+1,-q+3, \cdots q-3, q-1\}$ is the $k$-th target symbol sent from $S$ and $I(k) \in A$ is the $k$ th known interfering symbol sent from $R_{2} ; n(k)$ is the zero mean Gaussian noise with variance $\sigma^{2} ; h(k, l)$ and $h_{x}(k, l)$ are respectively the $l$-th tap channel coefficients from $R_{2}$ and $S$ to $R_{1}$ for their $k$-th symbols; $L_{x}$ and $L_{I}$ are the respective maximum tap delays of the two signals. The transmit powers, and the effects of transmit and receive pulse shapes, are combined into the channel coefficients, $h(k, l)$ and $h_{x}(k, l)$. According to the WSSUS (Wide-Sense Stationary-Uncorrelated Scattering) model of Bello [17], all the channel taps are independent of each other; for each tap, the channel variation satisfies $E\{h(k, l) h(k-1, l)\}=J_{0}\left(2 \pi f_{\max } \tau\right)$, where $J_{0}\left(2 \pi f_{\max } \tau\right)$ is the zeroth order Bessel function of the first kind, $f_{\max }$ is the maximum Doppler frequency, and $\tau$ is the symbol duration. Hereafter, the bold letter $\boldsymbol{x} / \boldsymbol{I}$ denotes the corresponding vector 
of the whole packet. Note that asynchrony between the signal and interference, if any, can be incorporated into the above baseband model. For example, if the symbols of the signal and interference are not aligned in time, the resulting sampling offset of the interference is equivalent to a multi-path effect that can be combined into the multi-path in (??). For carrier frequency asynchrony, the receiver can synchronize to the carrier frequency of the interference in a traditional way if we regard the target signal as "noise" during the BKIC process (the resulting frequency offset of the target signal, if any, can be processed after BKIC).

The system formulation for analog network coding in twoway relay channel is the same as in (??) and the details are omitted here.

\section{B. System Architecture}

We propose a blind known-interference cancellation scheme which can cancel the interference in (??) and transform $r(k)$ to the signal of interest, $\sum_{l=0}^{L_{x}} h_{x}(k, l) x(k-l)+n(k)$, plus a small noise as

$$
\begin{aligned}
& z(k)=\sum_{l=0}^{L_{x}} h_{x}(k, l) x(k-l)+n(k)+w(k) \\
& =x^{\prime}(k)+n(k)+w(k)
\end{aligned}
$$

where $w(k)$ is the residual interference introduced during the interference cancellation processing of our BKIC scheme. According to (??), we have the following two definitions:

Definition 1: Desired Signal (DS): $x^{\prime}(k)=$ $\sum_{l=0}^{L_{x}} h_{x}(k, l) x(k-l)$, which does not contain any noise or interference.

Definition 2: Desired Signal plus Noise (DSN): $x^{\prime}(k)+$ $n(k)=\sum_{l=0}^{L_{x}} h_{x}(k, l) x(k-l)+n(k)$, which is equivalent to the received signal from a pure point-to-point transmission without any interference. The function of BKIC is to remove the known interference. In the ideal case, its output should be exactly DSN. In reality, BKIC needs to estimate DSN as accurately as possible.

The signal $z(k)$ in (??) is DSN with a small extra noise, from which the traditional signal detection algorithm can then proceed to detect $\boldsymbol{x}$ as in conventional receivers. As will be shown later, the small noise $w(k)$ can be approximated as a Gaussian noise with negligible variance. Therefore, the transmission technique (i.e., modulation and other specifications) employed by source $S$ or the channel experienced by $S$ does not affect our BKIC scheme.

A relay enabled with our interference cancellation may be built as shown in the system architecture of Fig. 2. When the signal is received, the relay first checks if the known interference is present with the block "interference check". Interference check may be implemented with an optimal matched filter that projects the received signal to the locally known interference sequences $[19,20]$. In [19], a similar technique is used for spectrum sensing, in which instead of known interference, the signature of the primary user is known and is to be detected. As shown in the analysis and

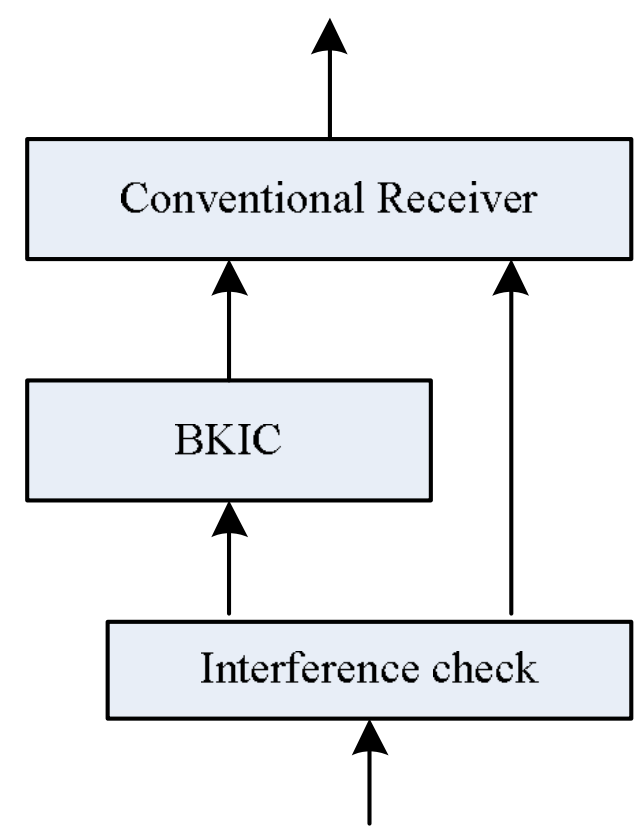

Fig. 2: System architecture with blind known-interference cancellation

experimental measurement in [19], the needed interference signal power for interference check decreases linearly with packet length and it could be as low as - $136 \mathrm{dbm}$. To limit the scope of this paper, we will not delve into the details of "interference check".

If known interference is not present, then the packet is directly fed to a conventional receiver for data detection. If known interference is present, then the packet is fed to the BKIC block to cancel the known interference. After that, the signal is fed to the conventional receiver for target data detection.

\section{BLIND KNOWN-INTERFERENCE CANCELLATION IN FLAT FADING CHANNEL}

In this section, we present our BKIC schemes assuming flat fading channel. The next section extends the treatment to the general multi-path channel. BKIC consists of two steps. In the first step, the interference is canceled by combining adjacent symbols. In the second step, the DSN, i.e., the pointto-point form of the signal, distorted during the cancellation step, is recovered. The second step is the non-trivial step, and we present two algorithms for it. The first algorithm, which serves as a benchmark, recovers DSN by means of smoothing; the second algorithm recovers DSN by a novel real-valued belief propagation framework.

\section{A. Step 1: Interference cancellation}

With flat fading, $L_{I}=1$ for the interference channel, we can rewrite (??) as

$$
r(k)=x^{\prime}(k)+h_{I}(k) I(k)+n(k) .
$$

In practice, the channel may be time varying, and the variation depends on the moving speed and other environmental factors. 
However, for adjacent symbols, the channel variation is very small. We can then approximate the channel variation as

$$
h_{I}(k+1) \approx h_{I}(k)+\Delta(k) .
$$

In (??), $\Delta(k) \sim N\left(0, \sigma_{\Delta}^{2}\right)$ is governed by the Doppler rate, and it is almost negligible in modern wireless communication systems [21, 25]. This key attribute enables us to use adjacent symbols to cancel the known interference without channel estimation. To do so, we obtain a new signal $t(k)$ by combining $r(k)$ and $r(k+1)$ as follows:

$$
\begin{aligned}
& t(k)=r(k)-\frac{I(k)}{I(k+1)} r(k+1) \\
& =\left(x^{\prime}(k)-\frac{I(k)}{I(k+1)} x^{\prime}(k+1)\right)+n(k) \quad k \in\{1,2, \cdots N-1\} \\
& \quad-\frac{I(k)}{I(k+1)} n(k+1)-I(k) \Delta(k)
\end{aligned}
$$

In (??), almost all the interference terms have been removed in $t(k)$. However, the signal of interest to us is DSN, $x^{\prime}(k)+n(k)$, rather than $t(k)$. In the next step, we show how to extract DSN from $t(k)$.

\section{B. Step 2: DSN recovery}

In (5), the target signal $x^{\prime}(k)$ is distorted into the form of $t(k)$ after the interference cancellation step. At first glance, $t(k)$ may appear to be the signal $x^{\prime}(k)$ passing through an Inter-Symbol Interference (ISI) channel, in which case traditional ISI equalization schemes such as filtering, Viterbi detection and Belief Propagation (BP) [22] detection, could be used to recover $x^{\prime}(k)$. However, a closer examination reveals an important difference between the signals $t(k)$ in (5) and that of a traditional ISI channel. Specifically, the difference is the correlated noise in (5) for adjacent symbols $t(k)$ and $t(k+1)$. Although Viterbi/BP detection achieves optimal MAP performance for independent noise in ISI equalization, its performance is far from optimal for the recovery of the target signal here because of the correlated noise, as will be shown in our numerical simulation.

Noise whitening is a standard technique for dealing with correlated noise. However, we cannot directly whiten the noise in (5) because it is impossible to transform the $N-1$ equations in (5) into $N$ equations with independent noise terms while maintaining the interference cancellation effect. Another possibility is the noise prediction and whitening process in [23]. As will be shown in our simulation results later, this scheme is also far from optimal.

We now propose two schemes to recover DSN with near optimal performance.

1) Recovery by Smoothing: From (5), we could write

$$
\begin{aligned}
& u(1)=t(1) \\
& =x^{\prime}(1)+n(1)-\frac{I(1)}{I(2)}\left(x^{\prime}(2)+n(2)\right)-I(1) \Delta(1) \\
& u(k)=u(k-1)+\frac{I(1)}{I(k)} t(k) \\
& =x^{\prime}(1)+n(1)-\frac{I(1)}{I(k+1)}\left(x^{\prime}(k+1)+n(k+1)\right) \\
& \quad-I(1) \sum_{m=1}^{k} \Delta(m) \quad \text { for } k \in\{2, \cdots N-2\}
\end{aligned}
$$

Then, we obtain the estimate of $x^{\prime}(1)+n(1)$ as follows:

$$
z(1)=\frac{1}{N-1} \sum_{k=1}^{N-1} u(k)=x^{\prime}(1)+n(1)+w(1)
$$

where the residual interference $w(1)=-\frac{1}{N-1}$ $\sum_{k=1}^{N-1}\left\{\frac{I(1)}{I(k+1)}\left(x^{\prime}(k+1)+n(k+1)\right)+I(1)(N-k) \Delta(k)\right\}$ is independent of $x^{\prime}(1)$ and $n(1)$. As will be shown in the next section, $w(k)$ can be approximated by Gaussian distribution. For slow fading ( $\sigma_{\Delta}^{2}$ almost equals 0 ), its variance is very small; for fast fading, it may become larger due to the accumulation of errors (note: this effect is inevitable in any channel estimation scheme). Then, we can remove $x^{\prime}(1)+n(1)$ from each signal in (5) to obtain the estimate of $x^{\prime}(k)+n(k)$ as:

$$
\begin{aligned}
& z(k)=\frac{I(k)}{I(1)}(z(1)-u(k-1)) \\
& =x^{\prime}(k)+n(k)+I(k)\left(w(1) / I(1)+\sum_{m=1}^{k-1} \Delta(m)\right) \\
& =x^{\prime}(k)+n(k)+w(k) \quad \text { for } k \geq 2
\end{aligned}
$$

We can then feed $z(k)$ to a conventional receiver for final desired data detection.

2) Recovery by Real-valued BP: Belief propagation is a powerful technique for inferring information from a large amount of correlated data. In the conventional method of applying BP to equalize ISI as in (5), $\boldsymbol{x}$ ' and its ISI form $x^{\prime}(k)-\frac{I(k)}{I(k+1)} x^{\prime}(k+1)$ are associated with the corresponding variable nodes. Their estimates are refined with message passing [22]. Direct BP application as such assumes the noise terms in the $N-1$ equations in (5) are independent. Strictly speaking, this is not true.

For correlated noise terms, [23] proposed to predict and whiten the correlated noise during the message passing procedure to improve the performance of the traditional BP algorithm. However, this method cannot make full use of the special form of noise correlation in (5) and can achieve only suboptimal performance. For better performance, we propose a novel BP scheme where DSN $\boldsymbol{x},+\boldsymbol{n}$ and the post-cancellation signal $\boldsymbol{t}$, rather than DS as in traditional BP, are associated with the variable nodes. Since they are real-valued signals, we refer to our BP detection algorithm as BKIC with Real-valued BP (BKIC-RBP). The Tanner graph of our BP algorithm is shown in Fig. 3.

An important subtlety in the Tanner graph is that $\boldsymbol{x}^{\boldsymbol{\prime}}+\boldsymbol{n}$ is treated as "signal symbols". With respect to Fig. 2, $\boldsymbol{x}$ ' $+\boldsymbol{n}$ is the target signal that will be fed to the conventional receiver after the interference cancelation process. With reference to (5), the observations $t(k)$ are made up of adjacent "signal symbols" plus noise in the cancellation process, which is $-I(k) \Delta(k)$ and does not include $n(k)$ and $n(k+1)$. The noise $n(k)$ will be dealt with by the conventional receiver later.

Remark: We stress that associating DSN rather than DS to the left variable node in Fig. 3 is the key of our BKICRBP scheme. First, directly estimating DS cannot improve the performance since the noise is independent of the interference. More importantly, $n(k)-\frac{I(k)}{I(k+1)} n(k+1)-I(k) \Delta(k)$ becomes 


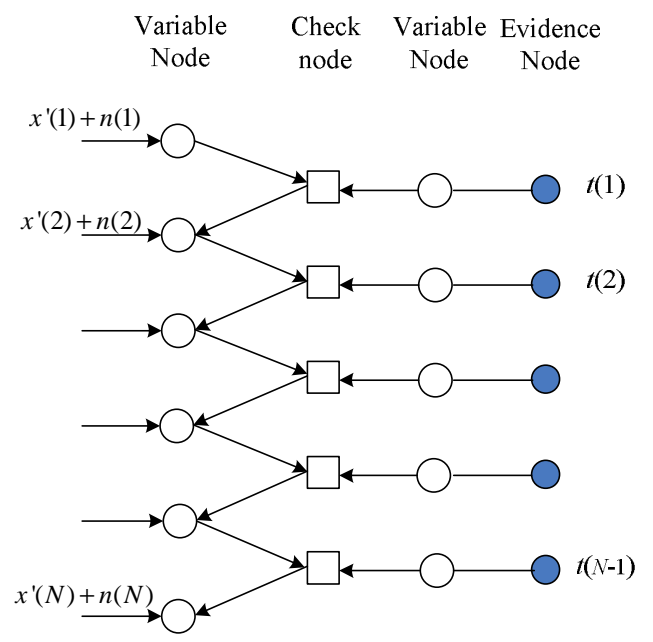

(a) Top-to-Bottom

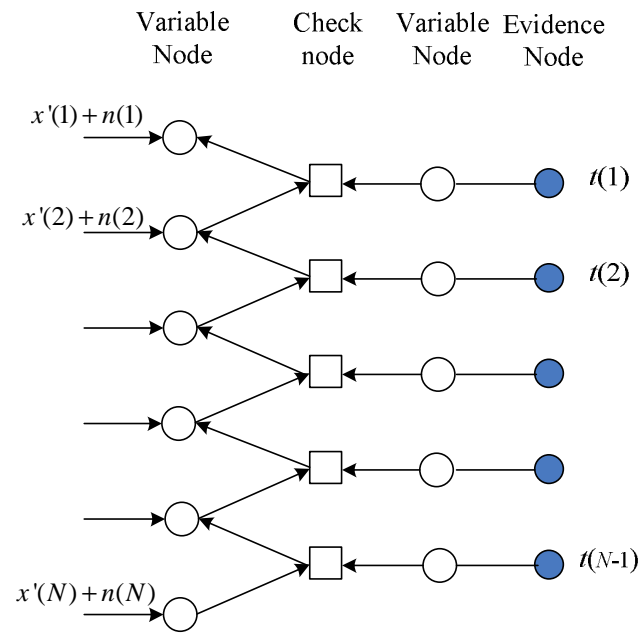

(b) Bottom-to-Top

Fig. 3: Tanner Graph for Continuous BP, where blank circles denote the variable nodes, the filled circles denote the evidence nodes and the rectangles denote the check nodes.

the general noise in $t$ and the relation between adjacent noise terms in (5) is wiped off from the figure when associating DS to the left variable node.

With the above setting, the target of BKIC-RBP is to find a vector $\mathbf{x}^{\prime}+\mathbf{n}$ to maximize

$$
\begin{aligned}
& P\left(\mathbf{x}^{\prime}+\mathbf{n} \mid \mathbf{t}\right) \propto P\left(\mathbf{t} \mid \mathbf{x}^{\prime}+\mathbf{n}\right) P\left(\mathbf{x}^{\prime}+\mathbf{n}\right) \\
& =\prod_{k} P\left(t(k) \mid x^{\prime}(k)+n(k), x^{\prime}(k+1)+n(k+1)\right) P\left(\mathbf{x}^{\prime}+\mathbf{n}\right) .
\end{aligned}
$$

Based on (9), a corresponding Tanner Graph can be established as in Fig. 3, where the messages being passed between the variable nodes and the check nodes are the probability density functions of the variable nodes at left hand side. The algorithm includes three critical steps: initializing the messages, updating the messages at the variable nodes and updating the messages at check nodes in an iterative way.

\section{Message initialization:}

The variable nodes $\boldsymbol{x}$ ' $+\boldsymbol{n}$ on the left side of Fig. 3 are not associated with any channel outputs, and we initialize the PDF of $\boldsymbol{x}^{\prime}+\boldsymbol{n}$ with the a priori probabilities. The variable $x^{\prime}$ adopts a discrete value, and the discrete distribution is determined by the constellation set and the multi-path channel. However, we cannot obtain the distribution because we assume the channel information is not yet available in BKIC (i.e., estimation of the signal channel, if any, occurs after BKIC as per the system architecture in Fig. 2). Generally speaking, the upper bound of the interference power, $P_{\max }$, can be derived easily. For example, $P_{\max }$ could be set to the max power of the received signal $\boldsymbol{r}^{1}$.

We assume $x^{\prime}$ is uniformly distributed in $\left[-\sqrt{P_{\max }}, \sqrt{P_{\max }}\right]^{2}$. Since the noise is of Gaussian distribution, the messages (i.e., the a priori probabilities) associated with the leftmost edges in the Tanner graph can be expressed as

$$
\begin{aligned}
& m_{x^{\prime}+n}=p_{x^{\prime}+n}(y)=p_{x^{\prime}+n}\left(x^{\prime}+n=y\right) \\
& =\int_{-\sqrt{P_{\max }}}^{\sqrt{P_{\max }}} p_{x^{\prime}}\left(x^{\prime}=s\right) p_{n}(n=y-s) d s \\
& =\frac{1}{2 \sqrt{P_{\max }} \sqrt{2 \pi} \sigma} \int_{-\sqrt{P_{\max }}}^{\sqrt{P_{\max }}} \exp \left(-(y-s)^{2} / 2 \sigma^{2}\right) d s
\end{aligned}
$$

For each left variable node $x^{\prime}(k)+n(k)$, there is an incoming edge from each of its adjacent check node, whose messages is denoted by $\overleftarrow{m}_{x^{\prime}(k)+n(k)}$, and an outgoing edge to the adjacent check node, whose message is denoted by $\vec{m}_{x^{\prime}(k)+n(k)}$.

With the initial messages, we then iteratively update them to obtain the final estimation. Since our Tanner graph in Fig. 3 does not include any cycles, one iteration is enough to obtain the optimal MAP performance. The iteration consists of two parallel message update processes. One process successively updates the top-to-bottom messages as illustrated in Fig. 3(a). That is, the right-bound message from the top $\vec{m}_{x^{\prime}(k)+n(k)}^{t 2 b}$ is first updated. Then the updated $\vec{m}_{x^{\prime}(k)+n(k)}^{t b}$ is used to update the next left-bound message $\overleftarrow{m}_{x^{\prime}(k)+n(k)}^{t 2 b}$, which is then used to update the next right-bound message, and so on and so forth. Similarly, the other process successively updates the bottomto-top messages, $\vec{m}_{x^{\prime}(k)+n(k)}^{b 2 t}$ and $\vec{m}_{x^{\prime}(k)+n(k)}^{b 2 t}$, as illustrated in Fig. 3(b). For each of the processes, the right-bound messages are messages going out of variable nodes, and the left-bound messages are messages going out of check nodes. These two types of messages are updated according to the variable-node update rule and the check-node update rule, respectively, in the following.

\section{Message updates at the check nodes:}

First consider the top-to-bottom process where the messages associated with the edges between the left variable nodes and the check nodes are updated one by one from top to bottom as in Fig. 3 (a). For a check node connected to the right evidence node $t[k]$, the two left variable nodes connected to it are $x^{\prime}(k)+n(k)$ and $x^{\prime}(k+1)+n(k+1)$. Given the input message $\vec{m}_{x^{\prime}(k)+n(k)}^{t 2 b}$, the output message can be calculated

\footnotetext{
${ }^{1} P_{\max }$ obtained in this way includes the power of DSN and the power of the interference. So it is a loose upper bound of the maximal interference power.

${ }^{2} \mathrm{~A}$ more accurate distribution of $x^{\prime}+n$ should improve the performance of BKIC-RBP. Fortunately, BKIC-RBP with this approximate a prior distribution still performs very well, as demonstrated by our simulation results later.
} 
using (5) as follows:

$$
\begin{aligned}
& \overleftarrow{m}_{x^{\prime}(k+1)+n(k+1)}^{t 2 b}=p_{x^{\prime}(k+1)+n(k+1)}(y)= \\
& \left|\frac{I(k+1)}{I(k)}\right| p_{x^{\prime}(k)+n(k)}\left(\frac{I(k) y}{I(k+1)}+t(k)+I(k) \Delta(k)\right)= \\
& \frac{I(k+1)}{I(k)} \mid \int_{\Delta(k)} p_{x^{\prime}(k)+n(k)}\left(\frac{I(k) y}{I(k+1)}+t(k)+I(k) s\right) p_{\Delta(k)}(s) d s \\
& \propto \int p_{x^{\prime}(k)+n(k)}\left(\frac{I(k)}{I(k+1)} y+t(k)+I(k) s\right) e^{-s^{2} / 2 \sigma_{\Delta}^{2}} d s
\end{aligned}
$$

where $\sigma_{\Delta}^{2}$ can be set to an upper bound of the variance of the interference term $\Delta(k)^{3}$. For block fading, $\Delta(k)=0$ with probability 1 . The PDF of $x^{\prime}(k+1)+n(k+1)$ in (11) can be simplified to

$$
\begin{aligned}
& \overleftarrow{m}_{x^{\prime}(k+1)+n(k+1)}^{t 2 b}=p_{x^{\prime}(k+1)+n(k+1)}(y) \\
& \propto p_{x^{\prime}(k)+n(k)}\left(\frac{I(k)}{I(k+1)} y+t(k)\right)
\end{aligned}
$$

Now consider the bottom-to-top process where the other set of messages associated with the edges between the left variable nodes and the check nodes are updated one by one from the bottom to the top as in Fig. 3 (b). Analogous to (11), the PDF of $x^{\prime}(k)+n(k)$ can be updated from the PDF of $x^{\prime}(k+1)+$ $n(k+1)$ and the observation $t(k)$ based on the following equation:

$$
\begin{aligned}
& \overleftarrow{m}_{x^{\prime}(k+1)+n(k+1)}^{b 2 t}=p_{x^{\prime}(k)+n(k)}(y) \propto \int e^{-s^{2} / 2 \sigma_{\Delta}^{2}} \\
& p_{x^{\prime}(k+1)+n(k+1)}\left(\frac{I(k+1)}{I(k)} y-\frac{I(k+1)}{I(k)} t(k)-I(k+1) s\right) d s
\end{aligned}
$$

For block fading, (??) can be simplified to

$$
\begin{aligned}
& \overleftarrow{m}_{x^{\prime}(k)+n(k)}^{b 2 t}=p_{x^{\prime}(k)+n(k)}(y) \\
& \propto p_{x^{\prime}(k+1)+n(k+1)}\left(\frac{I(k+1)}{I(k)} y-\frac{I(k+1)}{I(k)} t(k)\right)
\end{aligned}
$$

Message updates at the variable nodes:

Message updates at the left variable nodes is the same for both top-to-bottom process and the bottom-to-top process. Take the top-to-bottom processing as an example. Each left variable node is connected to three edges, whose associated messages are output message $\vec{m}_{x^{\prime}(k)+n(k)}^{t 2 b}$, input message $\overleftarrow{m}_{x^{\prime}(k)+n(k)}^{t 2 b}$ and the leftmost message with $m_{x^{\prime}+n}$ respectively. Each output message is updated with the two input messages as

$$
\begin{aligned}
& \vec{m}_{x^{\prime}(k)+n(k)}^{t 2 b}=p_{x^{\prime}(k)+n(k)}^{t 2 b}\left(y \mid \overleftarrow{m}_{x^{\prime}(k)+n(k)}^{t 2 b}, m_{x^{\prime}+n}\right) \\
& =\frac{1}{C} \overleftarrow{m}_{x^{\prime}(k)+n(k)}^{t 2 b} \cdot m_{x^{\prime}(k)+n(k)}
\end{aligned}
$$

where $C$ is a normalization factor.

At the end of the processing, we need to collect the information contained in all the messages and make a final estimate of DSN. For the $k$-th variable node with $x^{\prime}+n$, there is the $a$ priori PDF $p_{x^{\prime}(k)+n(k)}(y)$ contained in $m_{x^{\prime}+n}$, the extrinsic information on $p_{x^{\prime}(k)+n(k)}(y)$ contained in $\stackrel{\leftarrow}{m}_{x^{\prime}(k)+n(k)}^{t 2 b}$ after the top-to-bottom process, and another extrinsic information on $p_{x^{\prime}(k)+n(k)}(y)$ contained in $\overleftarrow{m}_{x^{\prime}(k)+n(k)}^{b 2 t}$ after the bottomto-top process (there is only one extrinsic PDF of the first

\footnotetext{
${ }^{3}$ BKIC-RBP is robust to the value of $\sigma_{\Delta}^{2}$ as shown in the simulation. Without an accurate estimate of $\sigma_{\Delta}^{2}$, we can fix it to a relative high value, such as 0.001 , with almost no performance loss.
}

and the last left variable node). Then the final probability distribution of $x^{\prime}(k)+n(k)$ can be calculated as the following product:

$$
\begin{aligned}
& p_{x^{\prime}(k)+n(k)}(y \mid t) \\
& =\frac{1}{C} m_{x^{\prime}(k)+n(k)} \cdot \overleftarrow{m}_{x^{\prime}(k)+n(k)}^{t 2 b} \cdot \stackrel{\leftarrow}{m}_{x^{\prime}(k)+n(k)}^{b 2 t}
\end{aligned}
$$

where $C$ is a normalization factor. $p_{x^{\prime}(k)+n(k)}(y \mid \mathbf{t})$ contains all the information about $x^{\prime}+n$ given the sequence $t$ after known interference cancellation and it can be fed to the traditional detection block for further target data detection. An estimate of $x^{\prime}+n$ is given by

$$
z(k)=\underset{y}{\arg \max } p_{x^{\prime}(k)+n(k)}(y \mid \mathbf{t})
$$

This estimate will be used in our numerical simulations later.

Discussion: we can regard $\boldsymbol{t}$ as an inner encoder output with input $x^{\prime}$. Then the decoder of it, i.e., the hard/soft decision based on $p_{x^{\prime}(k)+n(k)}(y \mid \mathbf{t})$ can be combined with the channel decoding procedure so that the Turbo like detection-decoding can applied to achieve even better performance.

\section{BKIC IN FREQUENCY SELECTIVE FADING CHANNEL}

The previous section considered the BKIC scheme for the flat fading channel. In this section, we extend the scheme to the frequency-selective channel. When BKIC is performed in a totally blind manner, we have no prior information about the multi-path characteristics. However, it is still reasonable to assume knowledge of the maximum delay of all the paths, $L$, as in many current broadband wireless systems. For example, the length of the predefined CP (cyclic prefix) in OFDM system implies the maximum delay of all paths. We can perform interference cancellation and recover the equivalent DSN for each potential path in a successive way as follows.

We first rewrite the received signal in (1) as

$$
\begin{aligned}
& r(k)=x^{\prime}(k)+\sum_{d=0}^{L} h_{I}(k, d) I(k-d)+n(k) \\
& =x^{\prime}(k)+\sum_{d=1}^{L} h_{I}(k, d) I(k-d)+h_{I}(k, 0) I(k)+n(k) \\
& =x_{0}^{\prime}(k)+h_{I}(k, 0) I(k)+n(k)
\end{aligned} .
$$

In (??), we select the first path of the interfering signal as the interference to be cancelled and combine the other interfering paths to DS, $x^{\prime}(k)$. Then, the selected interfering path can be removed by applying the BKIC scheme as in the preceding section. After that, we can obtain

$$
z_{1}(k)=x^{\prime}(k)+\sum_{d=1}^{L} h_{I}(k, d) I(k-d)+w_{0}(k)+n(k)
$$

where $w_{0}(k)$ is the residual interference after removing the first path.

Comparing $z_{1}(k)$ and $r(k)$, we see that the first path of interference has been removed and generated a new residual interference term $w_{0}$. In a similar way, we can remove the 
second path of interference to obtain $z_{2}(k)$. Repeat the BKIC scheme $L+1$ times, we finally obtain that

$$
z(k)=z_{L+1}(k)=x^{\prime}(k)+\sum_{d=0}^{L} w_{d}+n(k) .
$$

\section{Performance Analysis}

In this section, we analyze the BER and SINR performance of the proposed BKIC schemes under flat fading channels and frequency selective channels.

\section{A. BKIC under Flat Fading Channel}

Proposition 1: The performance of the overall system that incorporates the BKIC schemes as in Fig. 2 to cancel interference is upperbounded by the clean system where there is no interference at all.

This proposition is easy to understand since the interfering data is independent to the target data and it can not help to detect the target data. Due to the existence of the residual interference $w$, BKIC schemes can never achieve this upper bound exactly. Fortunately, we can approach it very closely.

Proposition 2: For the BKIC-S scheme, when the packet length $N$ is large, the residual interference $w(k)$ can be well approximated by a Gaussian noise $N(0, \mu(k))$, where the variance is $\mu(k)=I^{2}(k)\left(\frac{\left(P_{x}+\sigma^{2}\right)}{(N-1)}+\frac{N P_{I}}{3}\left(1-\alpha^{2}\right)\right) \mathrm{E}\left\{1 / I^{2}(j)\right\}$.

Proof: According to (8), we can show that the residual interference for the $k$-th symbol can be expressed as

$$
\begin{aligned}
& w(k)=I(k) \sum_{m=1}^{k-1} \Delta(m)- \\
& \frac{I(k)}{N-1} \sum_{j=1}^{N-1}\left\{\frac{1}{I(j+1)}\left(x^{\prime}(j+1)+n(j+1)\right)+(N-j) \Delta(j)\right\} \\
& =-I(k) \sum_{j=1}^{k-1}\left\{\frac{1-j}{N-1} \Delta(j)\right\}-I(k) \sum_{j=k}^{N-1}\left\{\frac{N-j}{N-1} \Delta(j)\right\} \\
& \quad-\frac{I(k)}{N-1} \sum_{j=1}^{N-1}\left\{\frac{x^{\prime}(j+1)}{I(j+1)}\right\}-\frac{I(k)}{N-1} \sum_{j=1}^{N-1}\left\{\frac{n(j+1)}{I(j+1)}\right\}
\end{aligned}
$$

In above expression of $w(k)^{4}$, all terms except the third are of the Gaussian distribution. Based on central limited theory, however, the third term can be well approximated as a Gaussian distribution for large $N$. Then, the total residual interference, $w$, can be regarded as of normal distribution. It is straightforward to verify that its mean value is zero and its variance is

$$
\begin{aligned}
& \mu(k)=\mathrm{E}\left\{|w(k)|^{2}\right\} \\
& =\frac{\left(P_{x}+\sigma^{2}\right)}{(N-1)} \mathrm{E}_{j}\left\{\frac{I^{2}(k)}{I^{2}(j)}\right\}+I^{2}(k) \sigma_{\Delta}^{2} \\
& \\
& {\left[\frac{(k-2)(k-1)(2 k-3)}{6(N-1)^{2}}+\frac{(N-k)(N-k+1)(2 N-2 k+1)}{6(N-1)^{2}}\right]}
\end{aligned}
$$

where $P_{x}$ is the received power of the target signal $x$ ' and $P_{I}$ is the received power of the interfering signal.

From the above proposition, we can obtain some important observations.

\footnotetext{
${ }^{4} \mathrm{By}$ checking the residual interference, we can find that BKIC-S is exactly equivalent to the traditional packet level KIC scheme: estimating the channel by an optimal filter matched to the known interference sequence and then subtracting the known interference.
}

Corollary 1: In BKIC-S, the residual interference is independent of the received power of the interfering signal for block fading channel.

In block fading channel, $\sigma_{\Delta}^{2}=0$. Then the power of the interfering signal, embedded in $h_{I}$, does not affect the performance of the BKIC-S scheme. This independence property is desired especially when the interfering signal power is much stronger than the desired signal. In contrast, the traditional known-interference cancellation [3] scheme, which is based on previously estimated channel information, performs poorly in this case because the residual interference is proportional to the interfering signal power with a given channel estimation mean square error (MSE).

Corollary 2: In BKIC-S, there is an optimal packet length ${ }^{5}$ $N$ that minimizes the residual interference $\mu$ for the continuous fading channel.

The first term in (??) decreases with $N$ as in the block fading channel. The second term in (??) increases with $N$. When $N$ is large, the channel varies far from its average value and the residual interference coming from channel variation accumulates (fortunately, BKIC-RBP is insensitive to channel variation.). If the interference data adopts a constant power modulation (PSK modulation), the optimal $N$ can be calculated as

$$
\partial \mu / \partial N=0 \quad \Rightarrow \quad N_{o p t}=1+\sqrt{\frac{3\left(P_{x}+\sigma^{2}\right)}{\sigma_{\Delta}^{2}}}
$$

In real communication systems, $\sigma_{\Delta}^{2}$ is very small and $N_{o p t}$ is large.

Besides the performance, complexity is also an important issue. The complexity of our BKIC-S scheme is quite low. Only one multiplication and two addition processes are needed for each symbol.

Corollary 3: With BKIC-S, the SNR loss compared to the clean system (only DSN signal exists) is

$$
\begin{aligned}
& \Delta=S N R_{D S N}-S N R_{B K I C-S} \\
& =10 \log \left(\frac{P_{x}}{\sigma^{2}}\right)-10 \log \left(\frac{P_{x}}{\sigma^{2}+\mu}\right) \\
& =10 \log \left(1+\frac{\mu}{\sigma^{2}}\right) \approx \mu S N R_{D S N}
\end{aligned}
$$

In BKIC-S, the residual interference is fixed when the power of DS plus noise is given. As a result, the SNR loss of BKIC-S depends on the SNR of DSN. For smaller residual interference, the SNR loss is approximately proportional to the SNR of DSN.

For the performance of BKIC-RBP, we have

Proposition 3: Given the assumption that $\Delta(k)$ for different $k$ are i.i.d Gaussian random variables, the performance of the BKIC-RBP scheme achieves the MAP optimal DSN recovery performance, which is lower bounded by the BKIC-S.

As is well known, the loop free BP detection has MAPoptimal performance. In our BKIC-RBP, there are no cycles in the Tanner graph in Fig. 3, so exact MAP performance of signal recovery can be achieved. As a result, the performance of BKIC-RBP is lower bounded by the BKIC-S scheme.

\footnotetext{
${ }^{5}$ In our paper, packet length is just the processing length of the algorithms. Dividing a packet into several parts for processing is possible but not considered here.
} 
Proposition 4: The complexity of BKIC-RBP is linear in terms of packet length.

The loop-free property in Fig. 3 also guarantees fast convergence of the $\mathrm{BP}$ algorithm. Only one iteration (one top-tobottom process and one bottom-to-top process) in the RBP algorithm is enough to obtain the MAP performance. The complexity of our RBP recovery is only $4 N-6$ message update operations, which is linear in terms of the packet length.

However, real value processing is needed to achieve the optimal MAP performance. In practice, we need to quantize the real valued PDF into discrete form with controlled complexity. As shown in our simulation and many other works [24], there is typically little performance loss associated with quantization errors in belief propagation algorithms.

\section{B. BKIC under Selective Fading Channel}

In multi-path channel, the flat fading BKIC is executed several times to successively get rid of the interference of each path. Therefore, the performance analysis is similar. We have

Proposition 5: For the BKIC-S in multi-path channel, the total residual interference $w$ can be approximated by a Gaussian noise $N\left(0, \sum_{m=0}^{L} \mu_{m}\right)$, where $\mu_{m}$ is the variance of the residual interference generated after cancellation of the $m$-th path. Its value can be obtained as in (21).

According to Proposition 5, we can obtain the corresponding corollaries as those in the flat fading channel.

Extending Proposition 3, we can obtain a similar proposition as follows:

Proposition 6: In the multi-path channel, BKIC-RBP achieves the MAP optimal performance for each path. Its performance is lower bounded by the performance of BKIC-S.

\section{NUMERICAL SimUlation}

This section presents numerical simulation results for the performance of BKIC. In our numerical studies, BPSK modulation is assumed for the desired signal while both BPSK and 4-ASK modulations are investigated for the interference signal. As is clear from the earlier discussion, the format of the target signal does not affect the operation of BKIC. Thus, without loss of generality in BKIC, we could assume flat fading with unit channel coefficient for the target-signal channel (note: with respect to Fig. 2, it is in the conventional receiver that the channel characteristics of the target signal that matters, and it is over there that the actual fading characteristics of the target signal channel come into play).

For the interference channel, block fading, continuous fading channel and multi-path fading are simulated. The system SNR is defined as $1 / \sigma^{2}$, where 1 is the power of the target signal and $\sigma^{2}$ is the variance of the noise. For BKIC-RBP, we need to quantize the messages (the PDFs) into discrete form to enable simulation with Matlab. In our simulation experiments, the quantization interval for BKIC-RBP is 0.025 for $1-7 \mathrm{~dB}$ and it is 0.0125 for $8-10 \mathrm{~dB}$. Quantization of the messages results in quantization errors, and smaller quantization step can further improve the performance at the cost of high complexity.

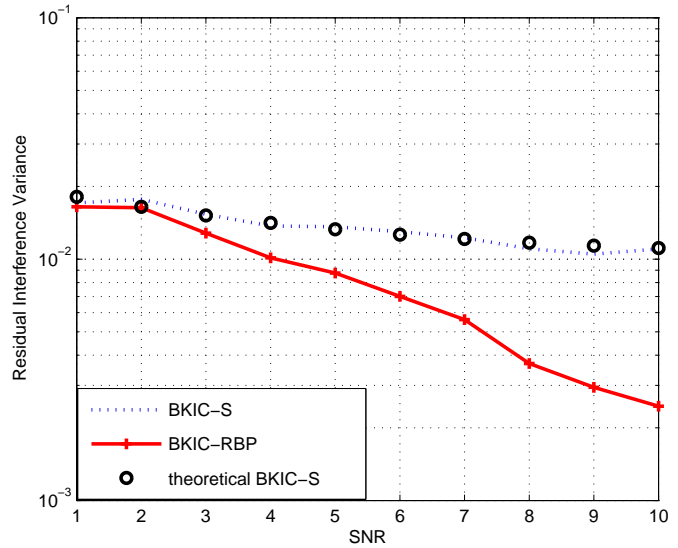

Fig. 4: Residual-interference variance with $\mathrm{N}=100$.

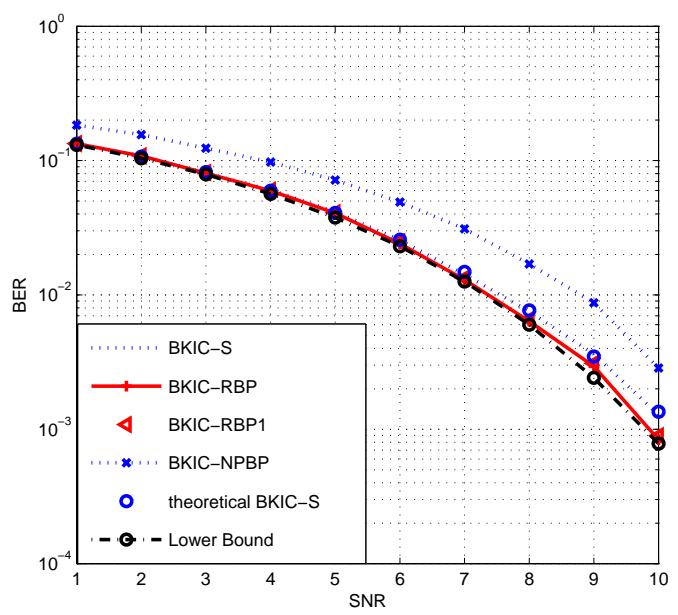

Fig. 5: BER performance for block fading with $\mathrm{N}=100$.

For comparison purposes, we also simulate the scheme of Noise Predictive Belief Propagation (NPBP) in [23] for the second step of BKIC. In our simulation, the length of the whitening filter in [23] is set to two. The complexity of BKIC-NPBP is $30(4 N-6)$ message update operations plus 3 whitening operations, which is much higher than BKIC-S and BKIC-RBP.

\section{A. Block Fading Channel}

We first consider the single path block fading interference channel. In this case, the channel coefficients are set to a constant unit within the whole block.

In Fig. 4 and Fig. 5, we show the variance of the residual interference and the BER of the proposed BKIC schemes respectively, with packet length $N=100$ (bits).

We first look at the simulation performance of the BKICS. For BKIC-S, the theoretical variance of the residual interference assuming Gaussian approximation is $\mu=\frac{\left(1+\sigma^{2}\right)}{(N-1)}$ according to (21). Based on it, we can calculate the theoretical BER of BKIC-S as $Q\left(\sqrt{1 /\left(\mu+\sigma^{2}\right)}\right)$ as in [25]. In both figures, the simulation results match the theoretical values very 


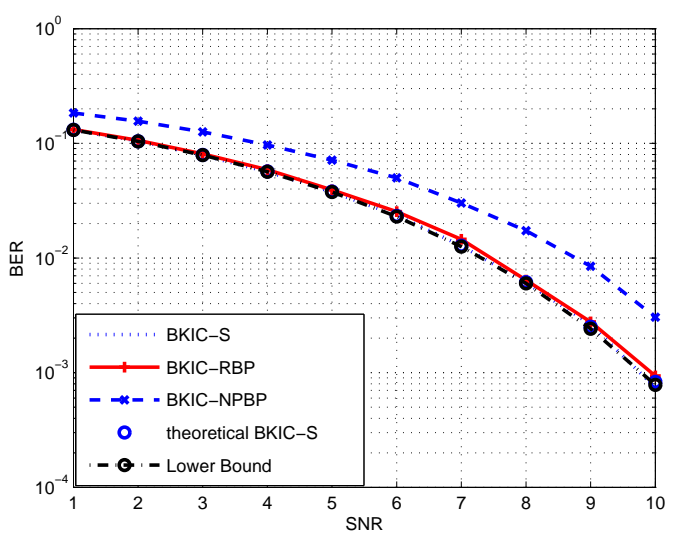

Fig. 6: BER performance for block fading with $\mathrm{N}=1000$.

well. This validates the Gaussian approximation in Proposition 2. In Fig. 4, we can see that the residual interference of BKIC$\mathrm{S}$ is almost independent of the SNR. The near-constant residual interference becomes more significant compared to noise in high SNR region. As a result, compared to the lower bound (i.e., the standard BPSK without interference), the SNR loss in Fig. 5 is larger when SNR increases. The SNR at BER of $2 \times 10^{-3}$ is around $10 \mathrm{~dB}$, corresponding to an SNR loss of more than $0.5 \mathrm{~dB}$.

As predicted by Proposition 3, the performance of BKICRBP, including residual interference and BER, is better than BKIC-S. The improvement becomes even larger in high SNR region. Both figures show that BKIC-RBP can benefit more from SNR increase. One possible reason is that high SNR will sharpen a priori distribution of DSN, which improves the performance of BKIC-RBP. By contrast, the conventional NPBP scheme is worse than BKIC-RBP by about $1.5 \mathrm{~dB}$. If we do not know whether the channel is block fading or continuous fading, and fixed $\sigma_{\Delta}^{2}$ to 0.001 , the performance of BKICRBP1 is obtained. The almost identical performance shows that BKIC-RBP is robust to $\sigma_{\Delta}^{2}$.

Fig. 6 shows the BER performances of different schemes for $N=1000$. As predicted in the analysis, the BER performance of BKIC-S improves significantly as $N$ increases. It is almost the same as the theoretical lower bound. On the other hand, the BER performance improvement of BKIC-RBP is negligible compared to the case of $N=100$ since the fixed quantization error dominates in the simulation.

In Fig. 7, we simulate the BKIC schemes by modulating the interference data with 4-ASK. For BKIC-RBP, the performance of 4-ASK is similar to that of BPSK. For BPSK-S and the traditional BKIC-NRBP, however, the performance of 4ASK is significantly degraded compared with that of BPSK. This can be explained by (21), from which we could see that the residual interference power is directly proportion to the interference power, 1 or 9 for 4-ASK. Therefore, half of the desired signal endures very larger residual interference, and that results in large BER like that caused by fading.

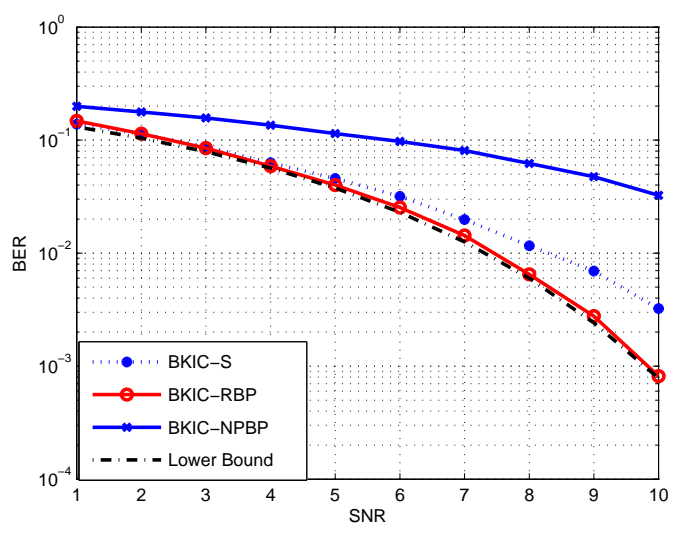

Fig. 7: BER performance for block fading channel, where the interference data is modulated with 4-ASK and $\mathrm{N}=100$.

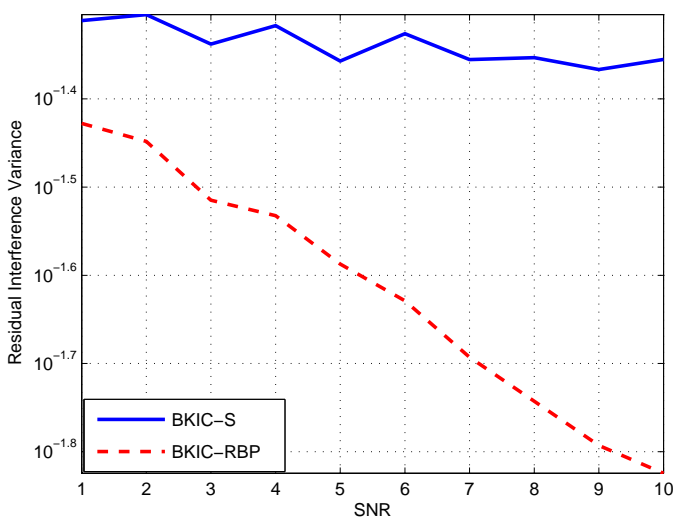

Fig. 8: Residual interference for continuous fading channel with $\mathrm{N}=100$.

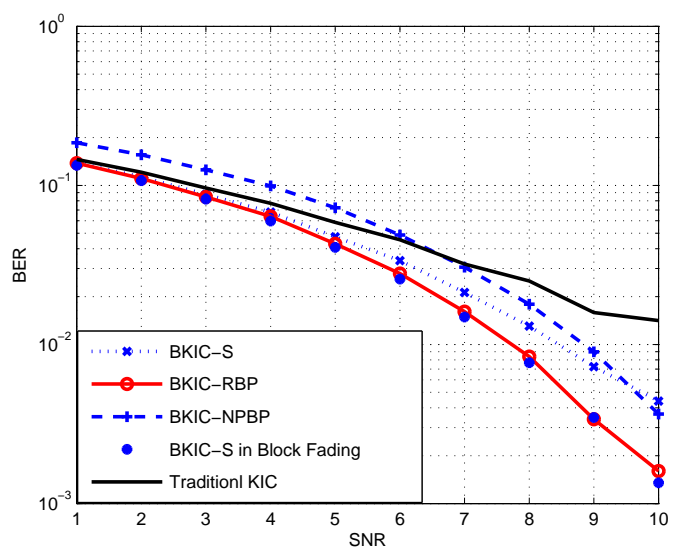

Fig. 9: BER performance for continuous fading channel with $\mathrm{N}=100$.

\section{B. Continuous Fading Channel}

In this part, we set the interference channel to single path continuous fading with the first order Markov channel mode in and the parameter $\alpha$ is set to $1-10^{-3}$ as in [18], which corresponds to a fast fading channel. $\sigma_{\Delta}^{2}$ is set to 0.001 . 


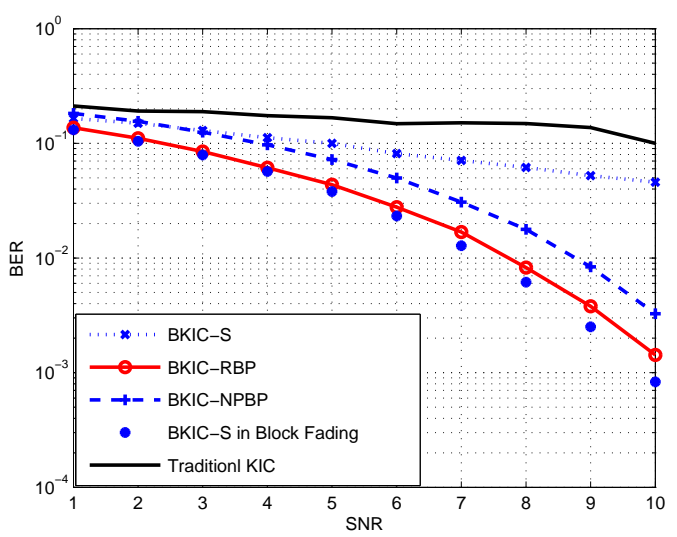

Fig. 10: BER performance for continuous fading channel with $\mathrm{N}=1000$.

Fig. 8 and Fig. 9 show the residual interference variance and the BER of the BKIC schemes with $N=100$, respectively. For comparison, we also give the BER performance of the traditional known-interference cancellation (Traditional KIC) scheme where the channel coefficient of the first symbol is perfectly known. Therefore, this performance is an upper bound of the actual Traditional KIC where there is always channel estimation error. From both figures, BKIC-RBP outperforms all the other schemes by at least $1 \mathrm{~dB}$. BKIC-S is the next best scheme when SNR is less than $10 \mathrm{~dB}$. Compared to Fig. 5, all schemes degrade when fading is continuous. The degradation for BKIC-RBP and BKIC-NPBP is about $0.1 \mathrm{~dB}$, which is much smaller than that of BKIC-S and traditional KIC.

In Fig. 10, we present the BER performance of the BKIC schemes when $N=1000$. With large $N$, the channel varies more significant and the BKIC-S scheme performs even worse. The BKIC-RBP scheme performs best among all the schemes and there is only $0.2 \mathrm{~dB}$ SNR loss compared to the block fading case. BKIC-RBP is about $1 \mathrm{~dB}$ better than the BKICNPBP scheme. It indicates that the BP algorithm is not significantly affected by channel variation.

\section{Frequency Selective Fading Channel}

We also investigate the BER performance of BKIC with frequency selective fading channel. The results are shown in Fig. 11. For illustration, a simple multipath interference channel scenario with two interference paths is considered. The amplitudes of the two paths are the same and unchanged within the packet; the delays of the two paths are 0 and 2 respectively. Compared to the flat fading case in Fig.5, both the performances of BKIC-RBP and BKIC-S are degraded as analyzed in the previous section. However, BKIC-S (traditional KIC) degrades more than BKIC-RBP, which has only tiny degradation.

\section{CONCLUSION}

This paper presents two known-interference cancellation schemes with good performance and low complexity. Although there has been much theoretical work in this area, deployments

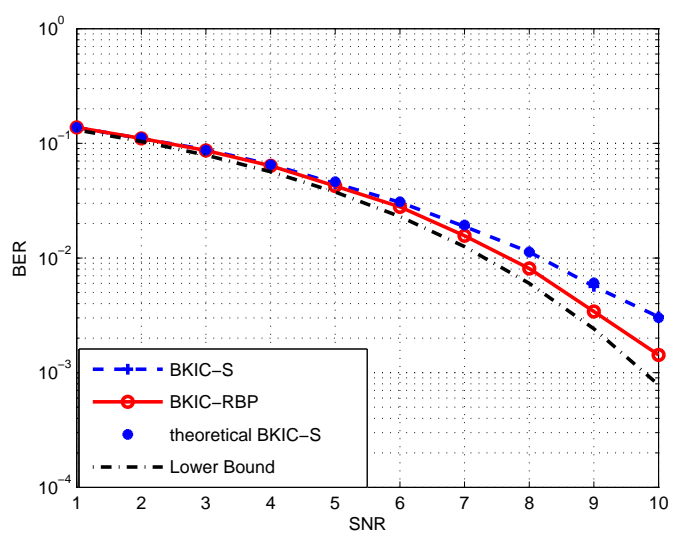

Fig. 11: BER performance for frequency selective fading channel with $\mathrm{N}=100$.

of the previously proposed schemes are difficult because of their needs for accurate channel estimation and their high complexity. To our knowledge, there has been no effective blind known-interference cancellation scheme that does not require estimation of the interference channel. Our work fills a gap in that regard.

Specifically, this paper proposes a framework for blind known-interference cancellation (BKIC), as embodied in Fig. 2. BKIC operates on the principle that the channel coefficient is almost constant for adjacent interference symbols in real communication systems. Thus, if the interference symbols are known, by combining adjacent symbols (i.e., combining the received signal and a weighted off-shifted version of it), we can obtain a new signal that is almost free of the interference. This, however, causes distortion to our target signal. A key challenge of BKIC, therefore, is how to compensate for this distortion. To tackle this challenge, we propose and investigate two schemes: BKIC-S, which is based on the principle of smoothing; and BKIC-RBP, which is based on the principle of real-value belief propagation. BKIC-RBP has MAP-optimal performance. The algorithmic complexities of both schemes are linear in the size of the packet.

We show that both BKIC-S and BKIC-RBP have superior performance compared with the traditional schemes. Importantly, their performance is very close to the theoretical performance bound, especially for block fading interference channel. The performance of BKIC-S depends on packet size, while the performance of BKIC-RBP is not sensitive to the packet size, but is dependent on the quantization step used in the algorithm to approximate real values. We also show that BKIC-RBP is very robust against fast fading in which the channel coefficients may vary in a dynamic manner within a packet.

Going forward, to fully exploit the potential of BKIC in wireless networks, new MAC layer and network layer protocols need to be designed. Besides relay networks, recently there has been increased interest in the wireless networking community on the realization of full-duplex wireless communication [26]. In the full duplex mode, a node transmits and receives at the same time. The received signal contains 
both the target signal as well as known interference (i.e., the self transmitted signal that is known). The investigation of BKIC for full-duplex wireless communication will be of much interest.

\section{ACKNOWLEDGMENT}

This work was partially supported by NSFC (No. 60209016), NSF Guangdong (No. 10151806001000003) and NSF Shenzhen (JC201005250034A, JC201005250047A). It was also supported by the General Research Fund (No. 414911), and the AoE grant E-02/08, established under the University Grant Committee of the Hong Kong Special Administrative Region, China.

The authors would like to thank Prof. Guo Dongning for his valuable discussion.

\section{REFERENCES}

[1] J. N. Laneman and G.W.Wornell, "Distributed space-time coded protocols for exploiting cooperative diversity in wireless networks," IEEE Trans. Inform. Theory, vol. 49, no. 10, pp. 2415-2425, Oct. 2003.

[2] G. Kramer, M. Gastpar and P. Gupta, "Cooperative strategies and capacity theorems for relay networks", IEEE Trans. Inform. Theory, 51 (9), pp. 3037-3063, September 2005.

[3] C. Qin, N. Santhapuri, S. Sen, S. Nelakuditi" Known Interference Cancellation: Resolving Collisions due to Repeated Transmissions" in Proc. Fifth IEEE Workshop on Wireless Mesh Networks (WIMESH 2010), June 2010.

[4] S. Zhang, S. Liew, and L. Lu "Physical layer network coding schemes over finite and infinite fields", Proc. IEEE Globecom 2008, Dec. 2008

[5] S. Katti, S. Gollakota, and D. Katabi, "Embracing Wireless Interference: Analog Network Coding”. Proc. ACM SIGCOMM, 2007.

[6] S. Boppana and J.M. Shea, "Overlapped transmission in wireless adhoc network," in International Conference on Communications, Circuits and Systems, Guilin, China, Jun. 2006, pp. 1309-1314.

[7] M. Weisenhorn and W. Hirt, "Robust noncoherent receiver exploiting UWB channel properties," in Proc. IEEE Ultrawideband Syst. Technol. (UWBST), Kyoto, Japan, May 2004, pp. 156-160.

[8] Q. Sun, D. C. Cox, and H. C. Huang, "Estimation of continuous flat fading MIMO channels," IEEE Trans. Wireless Commun., vol. 1, pp. 549-553, Oct. 2002.

[9] S. Zhang, S. Liew, and P. Lam, "Physical layer network coding", in Proc. ACM Mobicom 2006.

[10] L. Lu and S. C. Liew, "Asynchronous physical-layer network coding," http://arxiv.org/abs/1105.3144, May 2011, to appear in IEEE Trans. Wireless Commun.

[11] S. Liew, S. Zhang and L. Lu, "Physical layer network coding: tutorial, survey and beyond," to appear in Physical Communication, available at http://arxiv.org/abs/1105.4261.

[12] L. Lu, T. Wang, S. C. Liew, and S. Zhang, "Implementation of physicallayer network coding," to appear in Physical Communication, available at http://arxiv.org/abs/1105.3416.

[13] F. Gao, R. Zhang, and Y-C Liang, "On Channel Estimation for Amplifyand-Forward Two-Way Relay Networks" IEEE Transactions on Communications, Vol. 57, Issue 10, October 2009

[14] L. Song, Y. Li, A.Huang, B. Jiao, and A. V. Vasilakos, "Differential modulation for bidirectional relaying with analog network coding," IEEE Transactions on Signal Processing, vol. 58, no. 7, pp. 3933-3938, 2010.

[15] T. Cui, F. Gao, and C. Tellambura, "Differential modulation for two-way wireless communications: a perspective of differential network coding at the physical layer," IEEE Transactions on Communications, vol. 57, no. 10 , pp. 2977-2987, 2009.

[16] H. Rahul, H. Hassanieh, and D. Katabi, "Sourcesync: A cooperative wireless architecture for exploiting sender diversity," in Proc. ACM SIGCOMM, 2010.

[17] P. A. Bello, "Characterization of randomly time-variant linear channels," IEEE Trans. Commun. Syst., vol. CS-11, pp. 360-393, Dec. 1963.

[18] Y. Zhu, D. Guo and M. Honig, "A message-passing approach for joint channel estimation, interference mitigation, and decoding " IEEE Transactions on Wireless Communications, Vol 8(12), pp. 6008 - 6018, Dec. 2009.
[19] D. Cabric, A. Tkachenko, and R. Brodersen, "Spectrum sensing measurements of pilot, energy, and collaborative detection", in Proc. IEEE Milcom 2006 .

[20] S. Gollakota and D. Katabi, "ZigZag Decoding: Combating Hidden Terminals in Wireless Networks" in Proc. ACM SIGCOM 2008.

[21] W. C. Jakes Jr., Microwave Mobile Communications. New York: Wiley, 1974.

[22] M. N. Kaynak, T. M. Duman, and E. M. Kurtas, "Belief propagation over frequency selective fading channels," Proc. IEEE VTC'04-Fall, Sept. 2004, vol. 2, pp. 1367 - 1371.

[23] M. N. Kaynak, T. M. Duman, and E. M. Kurtas, "Noise predictive belief propagation," IEEE Transactions on Magnetics, vol. 41, no. 12, pp. 4427-4434, 2005.

[24] S.-Y. Chung, J. G. D. Forney, T. Richardson, and R. Urbanke, "On the design of low-density parity-check codes within $0.0045 \mathrm{~dB}$ of the Shannon limit," IEEE Commun. Lett., vol. 5, pp. 58-60, Feb. 2001.

[25] J. G. Proakis, Digital Communications. New York: McGraw Hill, 1995.

[26] J. Choi, M. Jain, K. Srinivasan, P. Levis, and S. Katti, "Achieving single channel, full duplex wireless communication" in Proc. ACM MobiCom 2010.

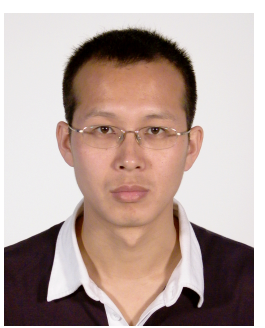

Shengli Zhang received his B. Eng. degree in electronic engineering and the M. Eng. degree in communication and information engineering from the University of Science and Technology of China (USTC), Hefei, China, in 2002 and 2005, respectively. He received the Ph.D. degree in the Department of Information Engineering, the Chinese University University of Hong Kong (CUHK), in 2008. From 2002 to 2005, he was with the Personal Communication Network and Spread Spectrum (PCNSS) Laboratory, USTC, as a Research Engineer involved in several National 863 Research Projects including the Beyond-3 Generation of Mobile System in China (FUTURE Plan). From 2002 to 2005, he was also a Research Engineer of the UTStarcom Wireless Soft Research Center, Hefei, China, involved in the research and implementation of the WCDMA communication systems. From Otc.2008, he was a Research Associate in CUHK. Now, he is an associate professor with the Communication Engineering Department, Shenzhen University, China. His current research interests include wireless networks, wireless communication, physical layer network coding, and cooperative wireless networks. 


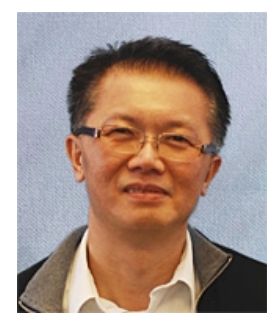

Soung Chang Liew received his S.B., S.M., E.E., and Ph.D. degrees from the Massachusetts Institute of Technology. From 1984 to 1988, he was at the MIT Laboratory for Information and Decision Systems, where he investigated Fiber-Optic Communications Networks. From March 1988 to July 1993, he was at Bellcore (now Telcordia), New Jersey, where he engaged in Broadband Network Research. He has been Professor at the Department of Information Engineering, the Chinese University of Hong Kong, since 1993. He is Adjunct Professor at Peking University and Southeast University, China.

Prof. Liews current research interests include wireless networks, Internet protocols, multimedia communications, and packet switch design. Prof. Liews research group won the best paper awards in IEEE MASS 2004 and IEEE WLN 2004. Separately, TCP Veno, a version of TCP to improve its performance over wireless networks proposed by Prof. Liews research group, has been incorporated into a recent release of Linux OS. In addition, Prof. Liew initiated and built the first inter-university ATM network testbed in Hong Kong in 1993. More recently, Prof. Liews research group pioneers the concept of Physical-layer Network Coding (PNC).

Besides academic activities, Prof. Liew is also active in the industry. He cofounded two technology start-ups in Internet Software and has been serving as consultant to many companies and industrial organizations. He is currently consultant for the Hong Kong Applied Science and Technology Research Institute (ASTRI), providing technical advice as well as helping to formulate $\mathrm{R} \& \mathrm{D}$ directions and strategies in the areas of Wireless Internetworking, Applications, and Services.

Prof. Liew is the holder of eight U.S. patents and Fellow of IEEE, IET and HKIE. He currently serves as Editor for IEEE Transactions on Wireless Communications and Ad Hoc and Sensor Wireless Networks. He is the recipient of the first Vice-Chancellor Exemplary Teaching Award at the Chinese University of Hong Kong. Publications of Prof. Liew can be found in www.ie.cuhk.edu.hk/soung.

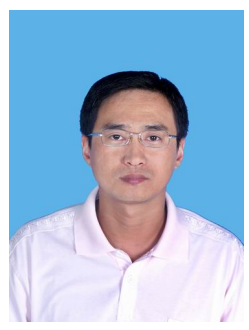

Hui Wang received his B.S., M.S. and Ph.D degrees from Xian Jiaotong University, in 1990, 1993, and 1996, respectively. He is now a professor in the College of Information Engineering, Shenzhen University. His research interests include wireless communication, signal processing, and distributed computing systems, in which, he is the author or co-author of more than 50 international leading journals, conferences and book chapters. 